\title{
"A Correlational Study to Assess the Umbilical Cord Coiling Index and Perinatal Outcomes in Selected Hospitals at Bijapur.
}

\author{
Ms. Afrinbanu Dyawapur \\ (Lecturer, Dept. Of OBG Nursing) BLDEA's Shri B M Patil Institute of Nursing Sciences, Bijapur, Karnataka, \\ India
}

\begin{abstract}
Umbilical cord, a narrow cord of tissue that connects a developing foetus with the placenta. Umbilical cord abnormalities are numerous, ranging from false knots to vasa pravia, which often leads to foetal death. An abnormal umbilical cord coiling index includes both hypo coiled cords (cords with an umbilical cord coiling index which is <10th percentile) and hyper coiled cords (cords with an umbilical cord coiling index which is $>90$ th percentile). As per many researchers, an abnormal umbilical coiling index has been reported to be related to adverse perinatal outcomes. The study was conducted in BLDEA's hospital and research centre, Bijapur. The sample consisted of 40 intranatal mothers who are admitted in labour ward and they were selected by Non random purposive sampling technique. An observational checklist was used to assess perinatal outcomes such as amniotic fluid, weighing machine to weigh the newborn APGAR score sheet to find the APGAR score of newborn. BALLARD score sheet to assess maturity of the baby in relation to Umbilical cord coiling Index Umbilical cord coiling was calculated among 40 intranatal mothers. The result were Hyper coil 12 (30\%), Normo coil 16 (40\%), Hypo coil $12(30 \%)$ and perinatal outcomes such as LBW, Meconium stained amniotic fluid, Low Apgar score and IUGR were found. The study reveals that there is a significant correlation between Umbilical cord coiling index and perinatal outcomes.
\end{abstract}

Keywords: Hyper coil, Hypo coil, Normo coil, umbilical cord coiling index

\section{Introduction}

Umbilical cord, derived from Latin word Funiculus Umbilical is, a narrow cord of tissue that connects a developing embryo, or fetus, with the placenta. ${ }^{1}$ One important feature of the umbilical cord is its coiling index (UCI). ${ }^{2}$ Abnormalities of cord coiling are coiling index, are Hypo coiling and hyper coiled cord and are associated with adverse outcome such as increased incidence of IUGR, LBW, oligo hydramnios, fetal anomalies, intrapartum fetal heart rate decelerations, meconium stained amniotic fluid, and low Apgar scores and Fetal demise etc. ${ }^{3}$ It can be concluded that the majority of pregnancies with over coiled or under coiled umbilical cords, as determined by assessment of UCI, have a normal outcome. However, there appear to be consistent and clinically significant associations between abnormal coiling and a range of adverse pregnancy outcomes. ${ }^{4}$

\subsection{Rationale}

The most significant anatomical structure of the umbilical cord is its level of coiling. ${ }^{5}$ Many studies show the crucial role played by the chemical composition of the Wharton's jelly in cord coiling, especially the role of a compound called hyaluronan. Hyaluronan aids in the growth of the umbilical vessels and subsequent coiling. ${ }^{6}$ These gradients may have an adverse effect on the development of the fetus in cases with over coiling (OC) or under coiling (UC) characteristics. ${ }^{5}$

After delivery usually umbilical cord is regarded as a waste and they are discarded. Where as if it is properly and accurately assessed it would render a significant indicator for the fetal well being and could be a tool for preventive perinatology. When umbilical cord coiling index is calculated antenatally through USG, results of many researches showed that UCI is significantly associated and correlated with perinatal outcomes which has equal weightage when UCI is measured postnatally for which limited data is available to its accuracy. Hence, the investigator felt to take up the study to measure the UCI postnatally and identifying the association between the index and perinatal outcome.

\subsection{Objectives}

$>$ To measure the umbilical cord coiling index (UCI) soon after the delivery.

$>$ To assess the perinatal outcomes.

$>$ To correlate the normo coiling, hypo coiling, and hyper coiling with perinatal outcome.

$>$ To find out the association between umbilical cord coiling index with selected demographic variables. 


\subsection{Hypothesis}

$\mathbf{H}_{1}$ : There will be significant correlation between hypo coiling and hyper coiling and perinatal outcomes

$\mathbf{H}_{2}$ : There will be significant association between umbilical cord coiling index and selected demographic variables.

\section{Supporting Literatures}

A study was conducted to correlate the perinatal outcome by noting the umbilical coiling index. In this study the umbilical cords of the babies born to 100 women were examined and umbilical coiling index was calculated. The study concludes that the hypo coiled cords or UCI $<10^{\text {th }}$ percentile is associated with the me conium staining, Apgar score at $1 \mathrm{~min}<4$ and Apgar score at $5 \mathrm{~min}<7$. The hyper coiled cord or UCI $>90^{\text {th }}$ percentile is associated with intra uterine growth restriction. ${ }^{7}$

A prospective study was conducted to evaluate the perinatal outcome with the abnormal umbilical cord coiling index. In this study 200 patients who were in active labour were included. The study concludes that hyper coiled cords or UCI which was $>$ 90th percentile was associated with IUGR and low ponderal indices. Hypo coiled cords or UCI which was $<10$ th percentile was associated with me conium staining, Apgar score at 1 min of $<4$ and at 5 min of $<7$, more LSCS rates and more NICU admissions.

A study was conducted at university college of medical sciences, Delhi to find out whether abnormal umbilical coiling index (UCI) is related to adverse antenatal and perinatal outcomes. In this study 107 umbilical cords were examined. The result shows that Low UCI was associated with adverse antenatal and perinatal complication

\section{Methodology}

1.1 Design : Descriptive correlational research design

1.2 Setting : BLDEA's Shri. B. M. Patil Medical Hospital \& Research Centre, Bijapur.

1.3 Participants : Intranatal mothers admitted for delivery in BLDEA's Shri. B. M. Patil Medical Hospital \& Research Centre, Bijapur.

1.4 Sample : 40 intranatal mothers who are admitted in labour ward.

1.5 Sampling Technique : Non random purposive sampling technique.

1.6 Tool used:

Socio-Demographic data :This part deals with demographic characteristics of intranatal mothers with their UCI and perinatal outcome, age, educational status, occupation, income of the family, religion, dietary pattern, residence. It includes 7 questions.

Observational check list: To assess the perinatal outcome in relation to number of coils.

- Check list for assessing amniotic fluid. It is used for assessing colour of amniotic fluid, clear indicates normal and Me conium stained indicates Foetal distress.

- Weighing machine to weigh the newborn. It is used to measure to weigh the newborn and classify as Extremely LBW, Very low birth weight, Low birth weight, Normal weight, Over weight

- APGAR score sheet to find the APGAR score of newborn as Severely depressed (0-3), Moderately depressed (4-6), Excellent condition (7-10)

- BALLARD score sheet to assess maturity of the baby such as Severe, Moderate, Mild and Normal

\subsection{Intervention}

$\checkmark$ Consent was obtained from the concerned authorities.

$\checkmark$ Purpose of conducting study was explained to the mother

$\checkmark$ Informed consent were obtained from the intranatal mothers

$\checkmark$ Umbilical cord will be measured from placental end to neonatal end

$\checkmark$ Umbilical cord coils will be counted

$\checkmark$ Amniotic fluid, Birth weight, APG AR score and BALLARD score sheet were used.

$\checkmark$ Perinatal outcomes were assessed by using the comparing chart.

\subsection{Data analysis}

- Socio-demographic variables of intranatal mothers-Frequency and $\%$ distribution of demographic characters, Chi-square analysis for association between UCI with demographic variables

- Correlation of Umblical cord coiling index and Perinatal outcomes-Mean, SD and Kuder's-20 test 


\section{Findings and Discussion}

Part A: Findings Related to the Demographic variables

- Majority of $13(32.5 \%)$ of the intranatal mothers were between the age group 18-22 years.

- Most $14(35.0 \%)$ of them were having primary education and $21(52.5 \%)$ of the intranatal mothers were house wives

- $12(30.0 \%)$ of the intranatal mothers were having family income of <3000 and majority of the intranatal mothers 34 (40\%) were Hindu.

- Most of the $13(32.5 \%)$ of the intranatal mothers were vegetarian and majority of $20(50.0 \%)$ of the intranatal mothers were belongs to rural area

Part B: Assess the perinatal outcomes relation to Umbilical cord coiling index

Table 1: Frequency and Percentage Distribution Of Pregnant Mothers According To UCI

\begin{tabular}{|l|l|l|}
\hline UCI & Frequency & Percent \\
\hline Hyper coiling & 12 & 30.0 \\
\hline Normo coiling & 16 & 40.0 \\
\hline Hypo coiling & 12 & 30.0 \\
\hline Total & 40 & 100.0 \\
\hline
\end{tabular}

Fig1: Frequency and Percentage Distribution of Pregnant Mothers According To UCI

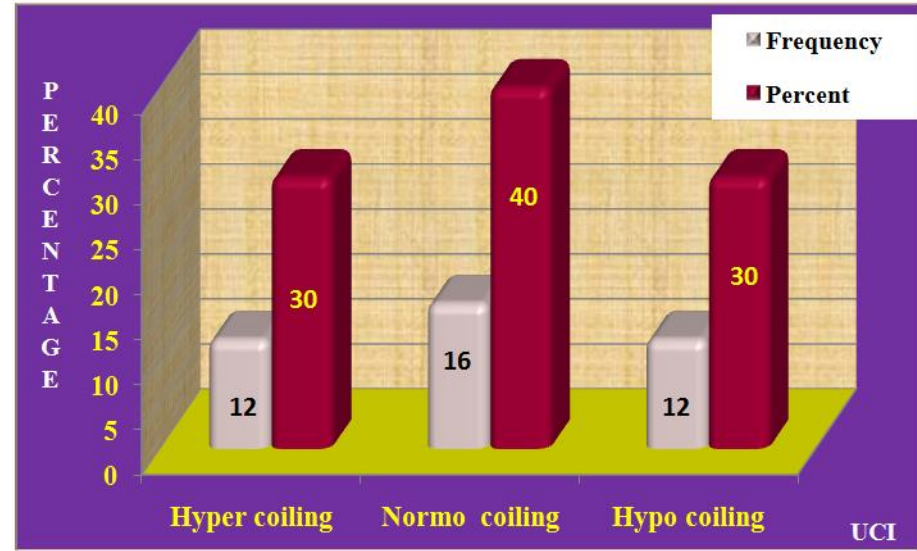

The above figure reveals that out of 40 samples, 12(30.0\%) of the intranatal mothers were having Hyper coils, 16(40.0\%) were having Normo coil, 12(30.0\%) were having Hypo coils.

Part C: Correlation of normo coiling, hypo coiling, and hyper coiling with perinatal outcomes.

Table 2: Frequency and Percentage Distribution of Pregnant Mothers According To Colour of Amniotic Fluid

\begin{tabular}{|c|c|c|c|c|}
\hline \multicolumn{5}{|c|}{ With Respect To UCI } \\
\hline Perinatal Outcome & UCI & & & \\
\hline Colour of Amniotic Fluid & Hypercoil & Normocoil & Hypocoil & Total \\
\hline Clear & 5 & 15 & 6 & 26 \\
\hline Meconium stained & 7 & 1 & 6 & 14 \\
\hline Total & 12 & 16 & 12 & 40 \\
\hline
\end{tabular}

Fig 2: Frequency and Percentage Distribution of Pregnant Mothers According To Colour of Amniotic Fluid With Respect To UCI

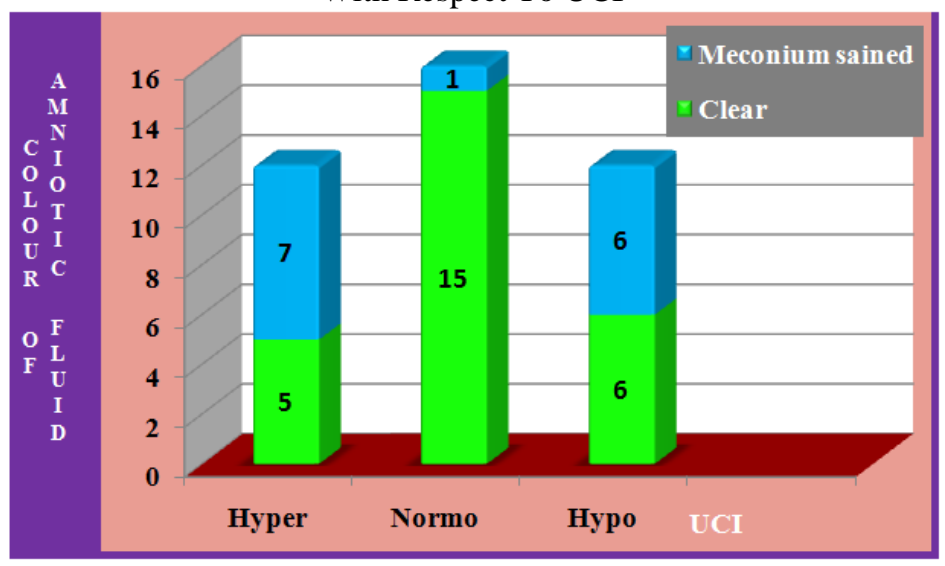


The above figure reveals that out of 26 clear amniotic fluid 5 were Hyper coil, 15 were Normo coil, and 6 were Hypo coil and out of 14 me conium stained amniotic fluid 7 were Hyper coil, 6 were Hypo coil and only 1 were Normo coil.

Table 3: Frequency Distribution of Pregnant Mothers According To Birth Weight With Respect To UCI

\begin{tabular}{|c|c|c|c|c|}
\hline Perinatal Outcome & UCI & & & \\
\hline Birth Weight & Hyper Coil & Norm Coil & Hypo Coil & Total \\
\hline Extremely Lbw & 3 & 14 & 2 & 19 \\
\hline Very Lbw & 6 & 2 & 6 & 14 \\
\hline Lbw & 3 & 0 & 2 & 5 \\
\hline Normal Weight & 0 & 0 & 2 & 2 \\
\hline Over Weight & 0 & 0 & 0 & 0 \\
\hline Total & 12 & 16 & 12 & 40 \\
\hline
\end{tabular}

Fig 3: Frequency Distribution of Pregnant Mothers According To Birth Weight With Respect To UCI

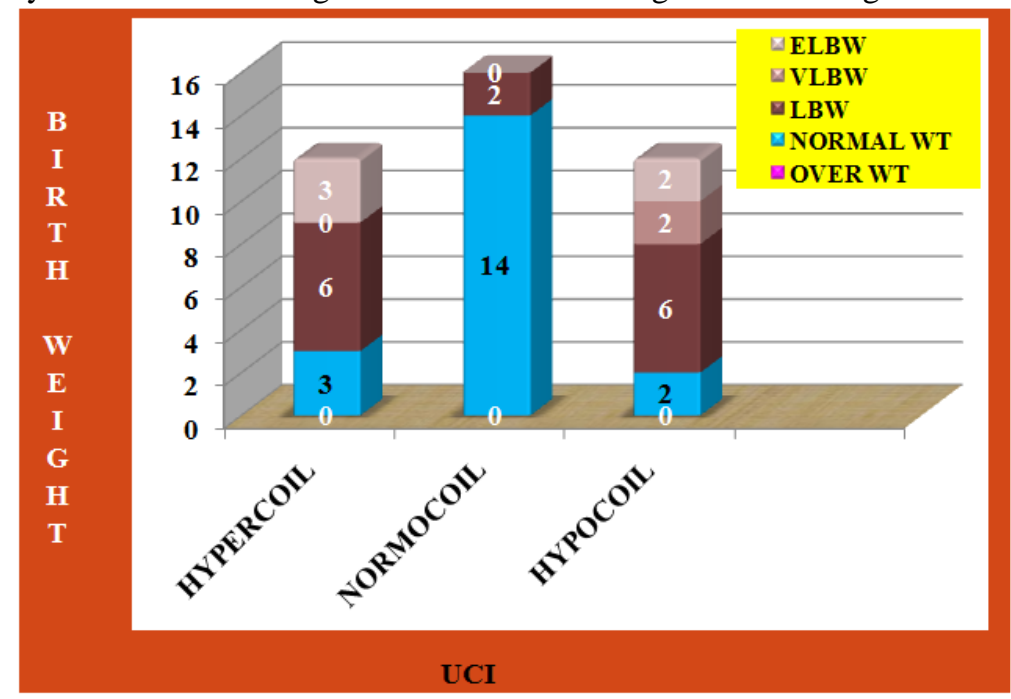

The above figure reveals that out of 40 samples, 5 babies were had extremely LBW in that 3 were hyper coil, 2 were hypo coil and no normo coils found. Out of 2 very LBW babies 2 were hypo coil and no hyper coil and hypo coil found. Out of 14 LBW babies 6 were hyper coil, 2 were normo coil and 6 were hypo coil. Out of 19 normal weight babies 3 were hyper coil, 14 were normo coil and 2 were hypo coil and no overweight found among 40 newborns

Table 4: Frequency Distribution of Pregnant Mothers According To 1min Apgar Score

\begin{tabular}{|l|l|l|l|l|}
\hline Perinatal Outcome & UCI & Total \\
\hline 1min Apgar Sore & Hypercoil & Normocoil & Hypocoil & Total \\
\hline Severe Depression 0-3 Score & 3 & 0 & 0 & 3 \\
\hline Moderately Depression 4-6 Score & 6 & 1 & 12 & 19 \\
\hline Excellent Condition 7-10 Score & 3 & 15 & 0 & 18 \\
\hline Total & 12 & 16 & 12 & 40 \\
\hline
\end{tabular}

Fig 4: Frequency Distribution of Pregnant Mothers According To 1min Apgar Score

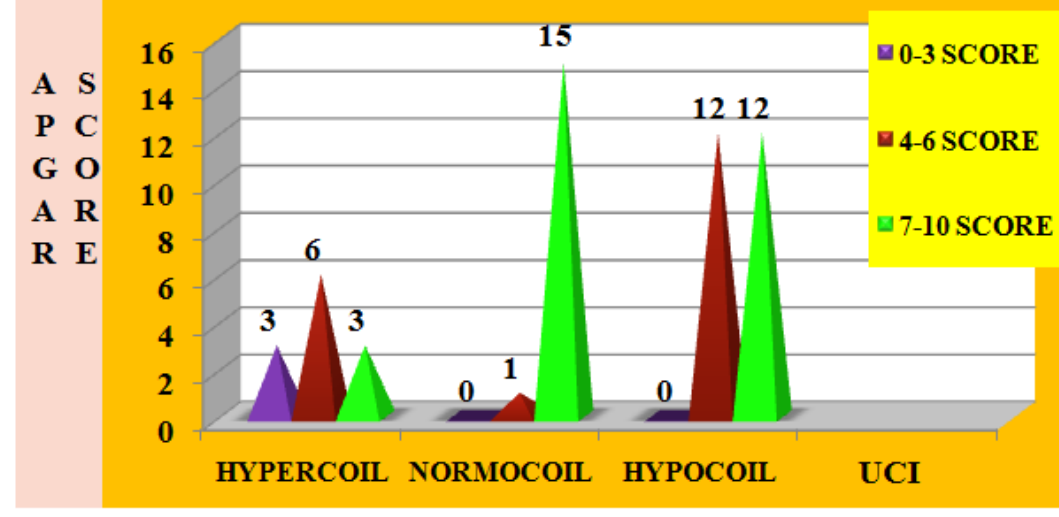


Out of 40 samples 3 were having 0-3 score at $1 \mathrm{~min}$, in that 3 were hyper coil and no hypo coil and normo coil found. 19 newborn were having 4-6 score in that 6 were hyper coil, 1were normo coil and 12 were hypo coil. 18 newborns were having 7-10 score in that 3 were hyper coil, 15 were normo coil and no hypo coil found.

Table 5: Frequency Distribution of Pregnant Mothers According To 5min Apgar Score

\begin{tabular}{|c|c|c|c|c|}
\hline Perinatal Outcome & UCI & & & \\
\hline 5min Apgar Score & Hyper Coil & Norm Coil & Hypo Coil & Total \\
\hline Severe Depression 0-3 Score & 3 & 0 & 0 & 3 \\
\hline Moderately Depression 4-6 Score & 1 & 0 & 8 & 9 \\
\hline Excellent Condition 7-10 Score & 8 & 16 & 4 & 28 \\
\hline Total & 12 & 16 & 12 & 40 \\
\hline
\end{tabular}

Fig 5: Frequency Distribution of Pregnant Mothers According To 5min Apgar Score

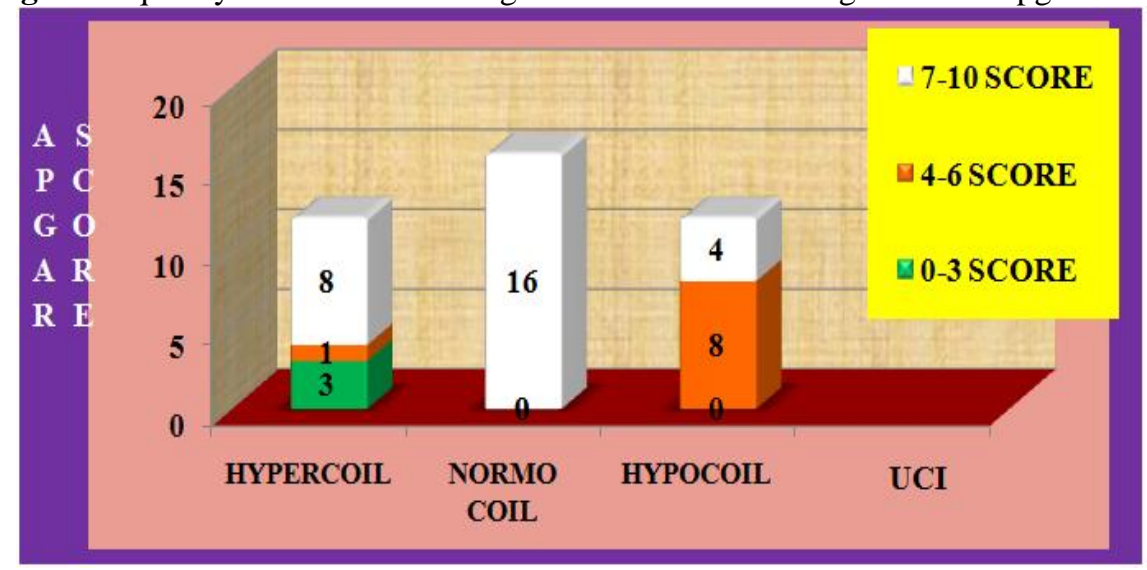

Out of 40 samples 3 were having 0-3 score at $5 \mathrm{~min}$, in that 3 were hyper coil and no hypo coil and normo coil found. 9 newborns were having 4-6 score in that 1 were hyper coil, 8 were hypo coil and no normo coil found. 28 newborns were having 7-10 score in that 8 hyper coil, 16 were normo coil and 4 were hypo coil.

Table 6: Frequency Distribution of Pregnant Mothers According To IUGR With Respect To UCI

\begin{tabular}{|l|l|l|l|l|}
\hline Perinatal Outcome & UCI & Norm coil & Hypo coil & Total \\
\hline Iugr & Hyper coil & 16 & 7 & 28 \\
\hline Normal & 5 & 0 & 2 & 5 \\
\hline Mild Iugr & 3 & 0 & 1 & 2 \\
\hline Moderate Iugr & 1 & 0 & 2 & 5 \\
\hline Severe Iugr & 3 & 16 & 12 & 40 \\
\hline Total & 12 & & \\
\hline
\end{tabular}

Fig 6: Frequency Distribution of Pregnant Mothers According To IUGR With Respect To UCI

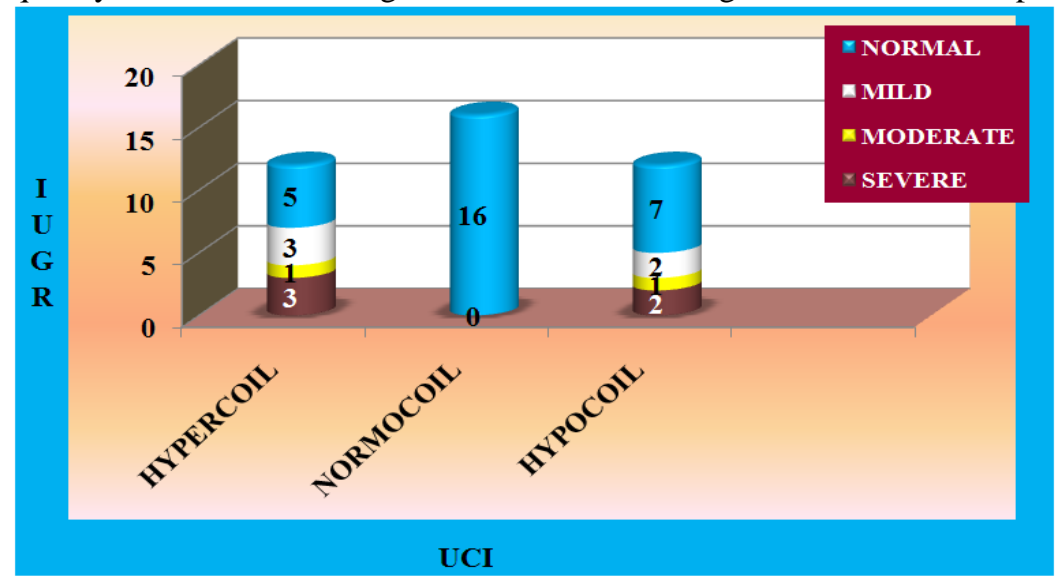

The data represented in the above figure depicts that out of 28 normal newborns 5 were hyper coil, 16 were normo coil and 7 were hypo coil. Out of 5 mild IUGR babies 3 were hyper coil 2 were hypo coil and no normo coil found. Out of 2 moderate IUGR 1 were hyper coil, 1 were hypo coil and no normo coil found. Out of 5 severe IUGR 3 were hyper coil, 2 were hypo coil and no normo coil found. 
“A Correlational Study To Assess The Umbilical Cord Coiling Index And Perinatal Outcomes In Selected...

Table 7: Frequency Distribution of Pregnant Mothers According To Gestational Age With Respect To UCI

\begin{tabular}{|l|l|l|l|l|}
\hline Perinatal Outcome & & UCI & & \\
\hline GESTATIONAL AGE & HYPERCOIL & NORMOCOIL & HYPOCOIL & TOTAL \\
\hline$<28$ WEEK & 1 & 0 & 1 & 2 \\
\hline 28-32 WEEK & 2 & 0 & 0 & 2 \\
\hline 33-37 WEEK & 4 & 3 & 11 & 18 \\
\hline$>$ 37 WEEK & 5 & 13 & 0 & 18 \\
\hline TOTAL & 12 & 12 & 40 \\
\hline
\end{tabular}

Fig 7: Frequency Distribution of Pregnant Mothers According To Gestational Age With Respect To UCI

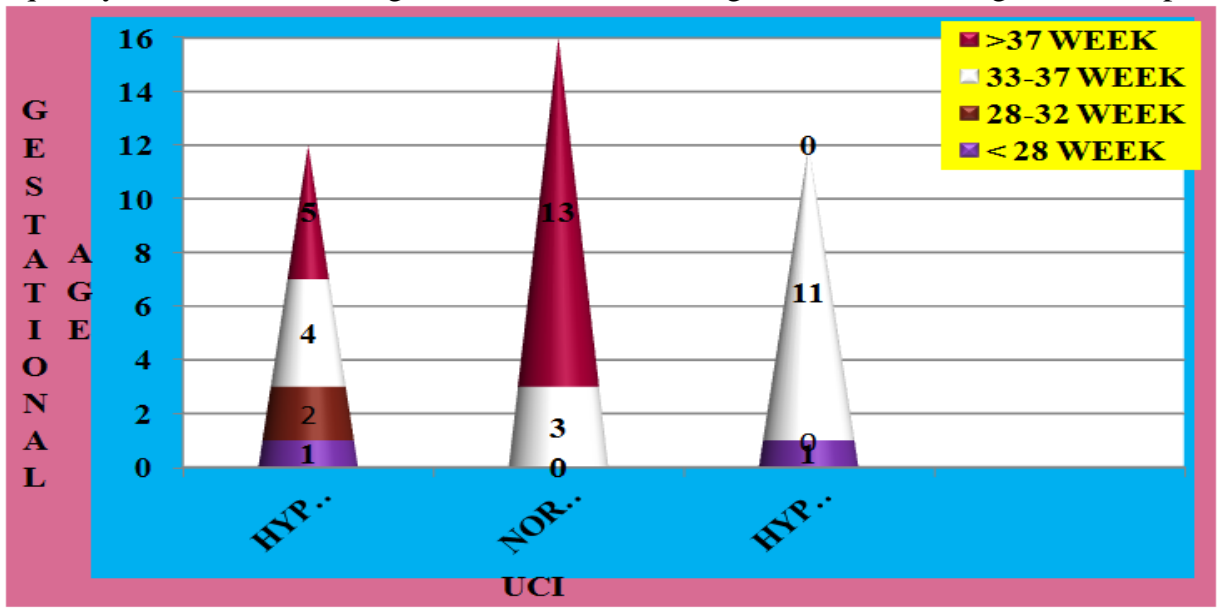

The data represented in the above figure depicts that out 40 intranatal mother 2 were having less than 28 weeks of gestation in that 2 were hyper coil, 1 were hypo coil and no normo coil found. Out of 2 the foetuses between 28-32 weeks of gestation 2 were hyper coil and no hypo coil and normo coil found. Out of 18 fetuses between 33-37 weeks of gestation 4 were hyper coil, 3 were normo coil and 11 were hypo coil. Out of 18 fetuses more than 37 weeks of gestation 5 were hyper coil, 13 were normo coil and no hypo coil found.

\section{Conclusion}

The umbilical cord is the fetal lifeline, which supplies the oxygen and nutrition needed, and transports the waste products from the fetus to the mother. . In Japanese folklore the umbilical cord has been called the "The flower stalk of life". One important feature of the umbilical cord is its coiling index (UCI), with hypo and hyper coiling being associated with Low Apgar score, fetal thrombotic vasculopathy (FTV), intolerance to labour, intrauterine growth restriction, LBW, cord stricture thrombosis of cord, Meconium stained amniotic fliud and chorionic blood vessels, and fetal demise. The coiled geometry of the umbilical cord largely affects umbilical blood flow that is vital for fetus's well-being and normal development.

Among 40 intranatal mothers the Umbilical cord coiling was calculated i.e. Hyper coil 12(30\%) Normo coil 16 (40\%), Hypo coil $12(30 \%)$ and perinatal outcomes such as LBW, Meconium stained amniotic fliud, Low Apgar score and IUGR were assessed. The study reveals that there is a significant correlation between Umbilical cord coiling index and perinatal outcomes. There is no significant association between any of the demographic variables with Umbilical cord coiling index

\section{Reference}

[1]. One important feature of the umbilical cord is its coiling index (UCI)

[2]. Peres LC, Taylor D. Overestimation of umbilical cord coiling index with segmental versus total length assessment. Journal of pediatric and Developmental Pathology. 2012 July-August; 15(4): 303-305.

[3]. www.fetalultrasound.com/online/abnormalities of cord coiling/34-068.htm

[4]. Sebire NJ. Pathophysiological significance of abnormal umbilical cord coiling index. Ultrasound Obstetrics and Gynecology. 2007; 30: 804-806. (www.interscience.wiley.com)

[5]. Aaron D, Kaplan, Ariel J. Jaffa, Ilan E.Timor. Hemodynamic Analysis of Arterial Blood Flow in the Coiled Umbilical Cord.Sage Journal.2010 November 2.

[6]. Joe Antony, Hypo coiled Umbilical Cord, OBGYN.net. 2011 September 8.

[7]. (Devaru Dakshayini,Thusoo Meghana.umbilical coiling index and perinatal outcome. The journal of obstetrics and gynecology of India. 2012 February; 62(1):63-66.)

[8]. (Nivedita S. Patil, Sunanda R. Kulkarni, Renu Lohitashwa. Umbilical Cord Coiling Index and Perinatal Outcome. Journal of Clinical and Diagnostic Research. 2013 August16; 7:1675-1677.

[9]. . ShaluGupta, Faridi MMA, Krishnan J. Umbilical coiling index. The journal of obstetrics and Gynecology of India.2006 July/August; 56(4): 315-319.) 